\title{
Contributed review: camera-limits for wide-field magnetic resonance imaging with a
} nitrogen-vacancy spin sensor

Wojciechowski, Adam M.; Karadas, Mursel; Huck, Alexander; Osterkamp, Christian; Jankuhn, Steffen; Meijer, Jan; Jelezko, Fedor; Andersen, Ulrik Lund

\section{Published in:}

Review of Scientific Instruments

Link to article, DOI:

$10.1063 / 1.5010282$

Publication date:

2018

Document Version

Publisher's PDF, also known as Version of record

Link back to DTU Orbit

Citation (APA):

Wojciechowski, A. M., Karadas, M., Huck, A., Osterkamp, C., Jankuhn, S., Meijer, J., Jelezko, F., \& Andersen, U. L. (2018). Contributed review: camera-limits for wide-field magnetic resonance imaging with a nitrogenvacancy spin sensor. Review of Scientific Instruments, 89(3), [031501]. https://doi.org/10.1063/1.5010282

\section{General rights}

Copyright and moral rights for the publications made accessible in the public portal are retained by the authors and/or other copyright owners and it is a condition of accessing publications that users recognise and abide by the legal requirements associated with these rights.

- Users may download and print one copy of any publication from the public portal for the purpose of private study or research.

- You may not further distribute the material or use it for any profit-making activity or commercial gain

- You may freely distribute the URL identifying the publication in the public portal 


\section{Contributed Review: Camera-limits for wide-field magnetic resonance imaging with a nitrogen-vacancy spin sensor}

Adam M. Wojciechowski, Mürsel Karadas, Alexander Huck, Christian Osterkamp, Steffen Jankuhn, Jan Meijer, Fedor Jelezko, and Ulrik L. Andersen

Citation: Review of Scientific Instruments 89, 031501 (2018); doi: 10.1063/1.5010282

View online: https://doi.org/10.1063/1.5010282

View Table of Contents: http://aip.scitation.org/toc/rsi/89/3

Published by the American Institute of Physics

\section{Articles you may be interested in}

Compact and tunable focusing device for plasma wakefield acceleration Review of Scientific Instruments 89, 033302 (2018); 10.1063/1.5006134

Experimental platform for investigations of high-intensity laser plasma interactions in the magnetic field of a pulsed power generator

Review of Scientific Instruments 89, 033504 (2018); 10.1063/1.5016973

Invited Review Article: Instrumentation for nuclear magnetic resonance in zero and ultralow magnetic field Review of Scientific Instruments 88, 091101 (2017); 10.1063/1.5003347

A $532 \mathrm{~nm}$ fiber-optic displacement interferometer for low-velocity impact experiments

Review of Scientific Instruments 89, 023101 (2018); 10.1063/1.4989767

Simple, low-noise piezo driver with feed-forward for broad tuning of external cavity diode lasers

Review of Scientific Instruments 89, 023102 (2018); 10.1063/1.5009643

Development of an electron momentum spectrometer for time-resolved experiments employing nanosecond pulsed electron beam

Review of Scientific Instruments 89, 033101 (2018); 10.1063/1.5018665

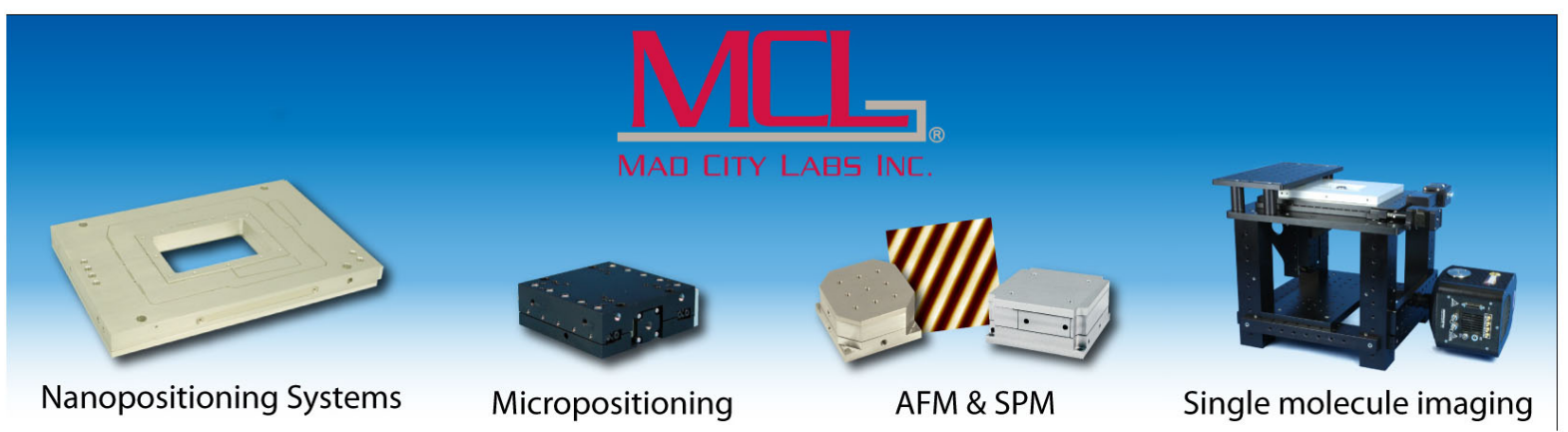




\title{
Contributed Review: Camera-limits for wide-field magnetic resonance imaging with a nitrogen-vacancy spin sensor
}

\author{
Adam M. Wojciechowski, ${ }^{1, a)}$ Mürsel Karadas, ${ }^{2}$ Alexander Huck, ${ }^{1}$ Christian Osterkamp, ${ }^{3}$ \\ Steffen Jankuhn, ${ }^{4}$ Jan Meijer, ${ }^{4}$ Fedor Jelezko, ${ }^{3}$ and Ulrik L. Andersen ${ }^{1}$ \\ ${ }^{1}$ Center for Macroscopic Quantum States (bigQ), Department of Physics, Technical University of Denmark, \\ 2800 Kongens Lyngby, Denmark \\ ${ }^{2}$ Department of Electrical Engineering, Technical University of Denmark, 2800 Kongens Lyngby, Denmark \\ ${ }^{3}$ Institute for Quantum Optics, Ulm University, Albert-Einstein-Allee 11, 89081 Ulm, Germany \\ ${ }^{4}$ Felix Bloch Institute for Solid State Physics, University of Leipzig, 04103 Leipzig, Germany
}

(Received 24 October 2017; accepted 24 February 2018; published online 26 March 2018)

\begin{abstract}
Sensitive, real-time optical magnetometry with nitrogen-vacancy centers in diamond relies on accurate imaging of small $\left(\ll 10^{-2}\right)$, fractional fluorescence changes across the diamond sample. We discuss the limitations on magnetic field sensitivity resulting from the limited number of photoelectrons that a camera can record in a given time. Several types of camera sensors are analyzed, and the smallest measurable magnetic field change is estimated for each type. We show that most common sensors are of a limited use in such applications, while certain highly specific cameras allow achieving nanoteslalevel sensitivity in $1 \mathrm{~s}$ of a combined exposure. Finally, we demonstrate the results obtained with a lock-in camera that paves the way for real-time, wide-field magnetometry at the nanotesla level and with a micrometer resolution. Published by AIP Publishing. https://doi.org/10.1063/1.5010282
\end{abstract}

\section{INTRODUCTION}

Over the recent years, there has been a surging interest in applications of the negatively charged nitrogen-vacancy (NV) color centers in diamond ${ }^{1,2}$ to precision sensing of temperature $^{3}$ and electromagnetic fields, ${ }^{4,5}$ owing to its high sensitivity per unit volume of such sensors. The smallest magnetic field sensors consist of a single NV center and provide nmscale spatial resolution, ${ }^{6}$ while the large bulk diamond sensors typically allow for sensing at micro- and millimeter length scales. A thin layer of $\mathrm{NV}$ centers, engineered close to the surface of a diamond, ${ }^{7,8}$ may be used for two-dimensional mapping of the magnetic field by projecting the NV fluorescence onto a camera sensor. ${ }^{9}$ Spatial resolution in 2D mapping depends on the imaging system and sensor parameters. Submicrometer resolution can be achieved with high magnification and high numerical-aperture (NA) microscopes equipped with a common CCD/CMOS ${ }^{10}$ camera. ${ }^{9,11}$ Wide-field imaging techniques were recently used to measure fields generated by magnetotactic bacteria, ${ }^{12}$ thin films, ${ }^{13}$ and magnetic beads, ${ }^{14}$ and to reconstruct the current flow inside integrated circuits ${ }^{15}$ or graphene sheets. ${ }^{16}$

Until now, wide-field magnetic field imaging was restricted to steady fields, with multiple (typically hundreds or more) image frames processed in order to fit the optically detected magnetic resonance (ODMR) spectrum with a sufficient signal-to-noise ratio (SNR). Complete spectral analysis provides full information on the magnetic field vector, at the cost of long measurement and post-processing times. ${ }^{11,17} \mathrm{In}$ this work, we focus on real-time imaging of small magnetic field changes that shift the ODMR resonance by a small fraction of its linewidth. By tuning the microwave (MW) source

a) Current address: Institute of Physics, Jagiellonian University, Łojasiewicza 11, 30-348 Kraków, Poland to the side of one ODMR resonance, a projection of the magnetic field vector onto a certain spatial direction can be directly imaged with a time resolution set by the camera frame rate. Since typical NV ensembles exhibit a fluorescence contrast of just a few percent, a camera sensor offering high SNR $\left(\gg 10^{2}\right)$ is required. Large SNR cameras have been recently developed for measuring small fluorescence variations of voltage sensitive dyes. ${ }^{18,19}$ Our approach utilizes a sensor originally developed for optical coherence tomography, ${ }^{20}$ which allows for phase-sensitive detection of a fluorescence signal and alleviates difficulties associated with low ODMR contrast.

In this article, we discuss the basic limitations for the NV diamond magnetometer sensitivity, which are imposed by the camera serving as a fluorescence detector. We compare several camera sensor types and the single-pixel sensitivity of a magnetometer using continuous wave (cw)-ODMR is estimated. The article is organized as follows: principles of magnetic field sensing using ODMR are described in Sec. II, the cameraimposed constraints are discussed in Sec. III, results of our experiments with a lock-in camera are presented in Sec. IV, and the findings are summarized in Sec. V.

\section{NV MAGNETOMETRY USING ODMR}

The most common scheme for camera imaging of dc and slowly varying magnetic fields with ensembles of NV centers relies on cw-ODMR. When the diamond sample is illuminated with green light, typically at a wavelength of around $532 \mathrm{~nm}$, a photoluminescence signal of the NV centers can be recorded in the $\sim 600$ to $800 \mathrm{~nm}$ spectral range at room temperature. Simultaneously, the NV centers are spin-polarized into the $m_{S}=0$ state within the $S=1$ ground state manifold, which provides higher fluorescence compared with the $m_{S}= \pm 1$ states. In the presence of a MW field resonant 
with the $m_{S}=0 \longleftrightarrow m_{S}= \pm 1$ transition, part of the $m_{S}=0$ population is transferred back to the $m_{S}= \pm 1$ state that is accompanied by the drop $\Delta F$ in the fluorescence level $F$. This is schematically shown in Fig. 1(a). Multiple MW resonances around the zero-field splitting of $\sim 2.8 \mathrm{GHz}$ can be observed in a typical ODMR spectrum corresponding to the four possible orientations of the NVs in a diamond matrix and two (three) nuclear spin projections (hyperfine structure) of the ${ }^{15} \mathrm{~N}\left({ }^{14} \mathrm{~N}\right)$ atom constituting the NV center. By aligning the magnetic field along certain crystal directions, some spin transitions may become degenerate, thereby reducing the overall number of resonances in the spectrum. This can be seen in Fig. 1(b), where the magnetic field is aligned with the diamond [110] direction, and hence only six resonances are visible.

The exact resonance frequencies in the ODMR spectrum are determined by a projection of the magnetic field vector onto the NV axis. By recording a complete spectrum with ensembles of NVs pointing along all four possible directions in the diamond matrix, full information on a magnetic field vector can be retrieved. However, if only a single component of the vector is of interest, the diamond sample can be oriented in a way that maximizes the field projection along a particular $\mathrm{NV}$ axis. This results in the largest resonance frequency shift for a given field change, i.e., highest magnetic field sensitivity. Apart from sample orientation, the magnetic field sensitivity depends on the contrast $C=\Delta F / F$ and linewidth $\Gamma$ of the observed ODMR resonance. Due to a finite difference in the fluorescence of $m_{S}=0$ and $m_{S}= \pm 1$ states and the fact that only a fraction of total NV ensemble is interacting with the MW field, the contrast is typically of the order $0.1 \%-1 \%$. Higher contrast $(>10 \%)$ can be observed in diamond samples where $\mathrm{NV}$ centers were preferentially aligned along one crystal direction during its growth. ${ }^{21,22}$ On the other hand, the resonance
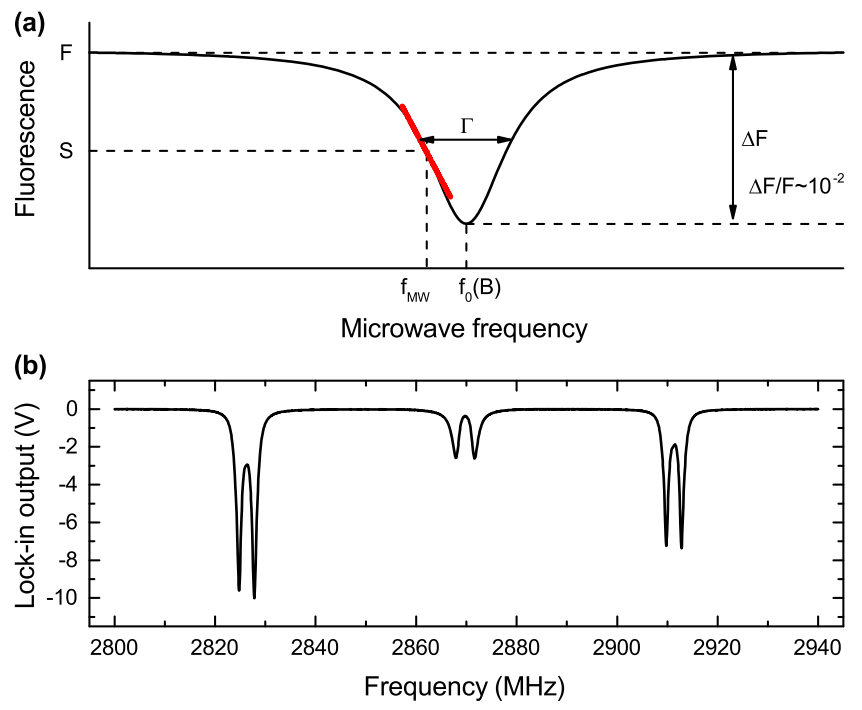

FIG. 1. (a) Principle of magnetic field measurement using ODMR. For a fixed microwave frequency $f_{M W}$, the fluorescence signal $S$ is recorded. Small changes in the magnetic field proportionally shift the MW resonance position $f_{0}$. Red line highlights the resonance slope that is used to map the detected fluorescence variations with the frequency shift. (b) ODMR spectrum of ${ }^{15} \mathrm{NV}$ recorded with a photodiode and amplitude-modulated MWs in the bias magnetic field of $\sim 2 \mathrm{mT}$ oriented in [110] direction. The peak depth corresponds to a contrast of $\sim 3 \%$. linewidth depends on the quality of the diamond sample and reflects its spin-dephasing time $T_{2}^{*}$. It is strongly affected by the concentration of ${ }^{13} \mathrm{C}$ and other impurities (including $\mathrm{N}$ and $\mathrm{NV}$ ) in the diamond lattice.

Fluorescence changes resulting from variations in the magnetic field may be recorded by tuning the MW frequency to the slope of the ODMR resonance [see Fig. 1(a)]. As the resonance frequency shifts, the observed fluorescence level changes proportionally to the resonance slope (red line). The sensitivity of such a measurement is fundamentally limited by the spin-projection noise:

$$
\delta B \simeq \frac{\hbar}{g \mu_{B}} \frac{1}{\sqrt{n_{0} V \tau T_{2}^{*}}},
$$

where $g$ is the Lande factor, $\mu_{B}$ is the Bohr magneton, $n_{0}$ is the $\mathrm{NV}$ density, $V$ is the sample volume, and $\tau$ is the measurement time, $\tau>>T_{2}^{*}$. We assumed here that the magnetic field direction is along the NV quantization axis. Equation (1) can be understood as an uncertainty resulting from performing independent measurements on an ensemble of $n_{0} \cdot V$ spins, repeated every $T_{2}^{*}$ time over the full measurement time of $\tau$. For values matching our diamond sample, an NV concentration of $[\mathrm{NV}]=1 \mathrm{ppm}$ and a dephasing time $T_{2}^{*}=1 \mu \mathrm{s}$, the resulting sensitivity limit is as low as $\delta B \sim 15 \mathrm{pT} \mathrm{Hz}^{-1 / 2}$ for a sensing volume of $1 \mu \mathrm{m}^{3}$. An order of magnitude higher NV concentrations reported in Ref. 23 and longer dephasing times observed in Ref. 24 indicate potential for further sensitivity enhancements. Although the increase of $\mathrm{N}$ and NV concentrations typically degrades $T_{2}^{*}$, the optimized sample fabrication process is believed to allow $T_{2}^{*}$ times as long as $30 \mu \mathrm{s}$ with $[\mathrm{NV}]=1 \mathrm{ppm} .{ }^{25}$

The spin-projection limit, given by Eq. (1), holds when the spin states are perfectly discernible. In cw-ODMR, however, only a limited contrast $C$ is observed. Additionally, the NV magnetometers suffer from a finite collection efficiency and transmission through the imaging optics, light trapping inside the diamond due to total internal reflection, and detector imperfections. It is thus sensible to express the sensitivity limit in terms of number of fluorescence photons,

$$
\delta B \simeq \frac{\hbar}{g \mu_{B}} \frac{\Gamma}{C \sqrt{\eta \mathcal{R} \tau}},
$$

where $\Gamma$ is the resonance linewidth, $\mathcal{R}$ is the NV fluorescence rate, $\eta$ is the photon collection efficiency, and we have omitted a prefactor of $0.77(0.7)$ stemming from the Lorentzian (Gaussian) shape of the resonance. The presence of contrast $C$ in the denominator accounts for the limited discernibility of the spin states by means of the fluorescence measurement. The observed resonance linewidth $\Gamma$ results from the power broadening due to the optical and microwave fields; however, for an optimized magnetometer, this value is in the order of $1 / T_{2}^{*}$.

Many optical detectors, including most camera sensors, are subject to saturation and impose a limit on the number of photons that can be recorded in a given time. In this case, it is practical to formulate the sensitivity in terms of the actual signal, $S$, and noise, $N$, ratio in the recorded image, rather than the fluorescence rate: 


$$
\delta B \simeq \frac{\hbar}{g \mu_{B}} \frac{\Gamma}{C \cdot \mathrm{SNR}},
$$

where $\mathrm{SNR} \equiv S / N$. In general, the noise term consists of optical shot-noise $N_{\text {opt }}$ associated with the recorded fluorescence, electronic noise of the detector $N_{e l}$, and other technical contributions. For the sake of brevity, the technical noise (e.g., the instability of excitation-light power) is neglected here. For an optimized (shot-noise limited) detector, $N_{\text {opt }}$ becomes the dominating noise term, $N \approx N_{\text {opt }}$, and the signal-to-noise ratio is simply given by $\mathrm{SNR}=\sqrt{S}$. The magnetic field sensitivity can then be improved by increasing the number of photons that can be collected in a single measurement.

\section{SENSITIVITY LIMITS WITH CAMERA DETECTION}

The important ingredient for achieving high magnetic field sensitivity, as discussed above, is the ability of the imaging system to collect a large number of photons. In the case of NV imaging, this involves optimizing the delivery of excitation light, maximizing fluorescence collection efficiency and imaging optics throughput, and, finally, choosing a sensor capable of recording most of the incoming photons. The latter process is limited by the quantum efficiency (QE), the size of the photo-active area within a pixel [fill factor (FF); see Fig. 2(a)] and, most importantly, the finite amount of charge that can be stored in a pixel without signal degradation, referred to as the full well capacity (FWC). Below we discuss these parameters as they are important for highsensitivity, wide-field magnetic imaging. In order to support the analysis with realistic parameter values, we have analyzed several cameras with various sensor types and listed them in Table I.

\section{A. Full well capacity}

When the camera is overexposed, i.e., the number of photoelectrons exceeds the FWC, the recorded image becomes distorted. The exact signal degradation depends on the sensor technology and particular design. However, the main sources of distortion are the saturation of intensity recorded by a pixel and the leakage (bleeding) of the photoelectrons to the neighboring pixels, a process called blooming and affecting most CCD sensors. During a single exposure of the camera, the maximum number of photoelectrons (charges) captured by a pixel is $S_{\max }=$ FWC. This number is independent of the fluorescence collection efficiency $\eta$, exposure time $\tau$, pixel size, quantum efficiency (QE), and filling factor (FF) of the sensor itself. The optical shot-noise, $N_{o p t}$, associated with such a signal level also reaches the maximum value $N_{\text {opt, max }}=\sqrt{\mathrm{FWC}}$ and dominates other noise types. The readout noise is typically in the order of $1-10 \mathrm{e}^{-}$and is relevant only for low light levels, while the dark noise only affects long-exposure images. Therefore, the signal-to-noise ratio per pixel for a single exposure is equal to $\mathrm{SNR}_{p x}^{\exp } \approx \sqrt{S}$ and has a maximum value of

$$
\mathrm{SNR}_{p x, \text { max }}^{\exp } \approx \sqrt{\mathrm{FWC}}
$$

when the camera is fully exposed. Since each pixel can collect only up to FWC photoelectrons, Eq. (4) sets an upper
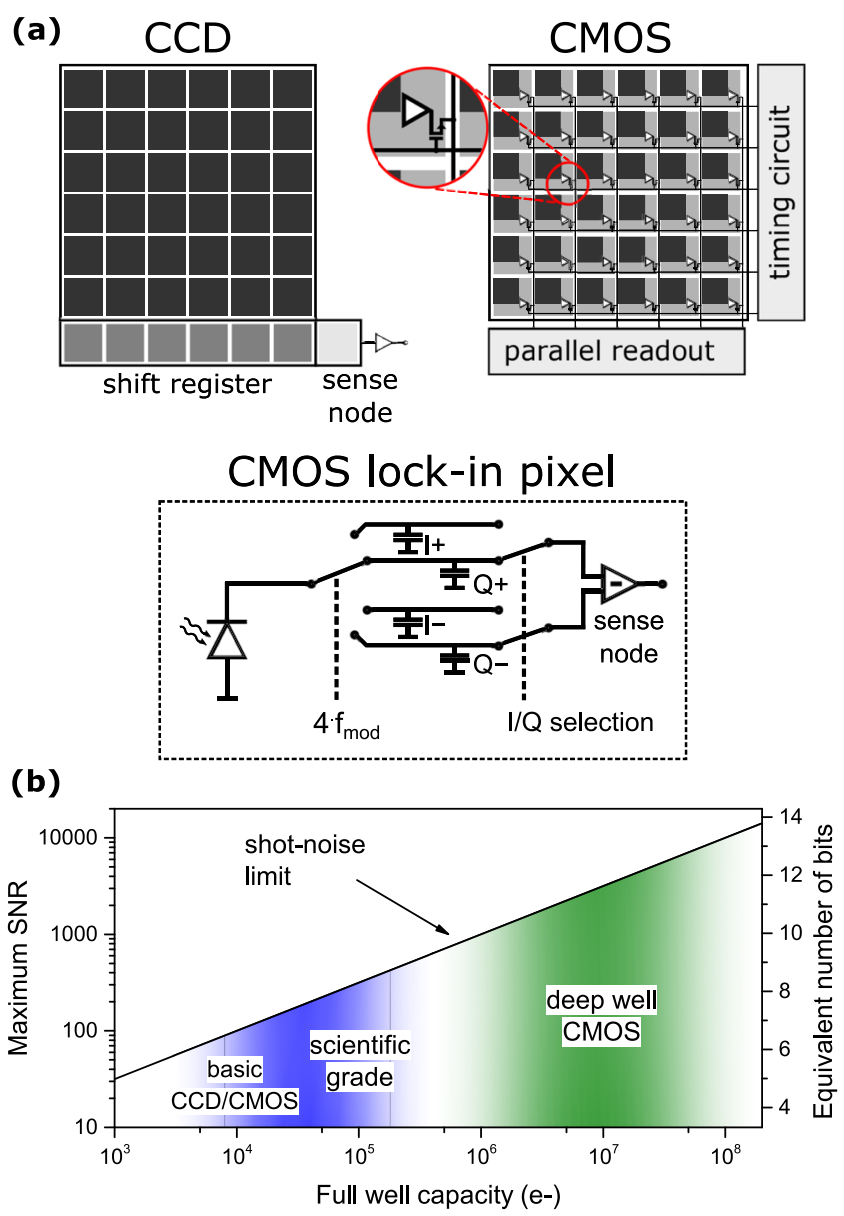

FIG. 2. (a) Illustrative diagram of camera sensor operation. Photo-active area is shaded black. For a CCD matrix, the collected charge is transferred downwards line-by-line to the shift register and then consecutively converted to a voltage and digitized. CMOS sensors use in-pixel transistors for charge conversion, which enables high-speed, random-access readout at the cost of a limited photo-active surface. Lock-in sensor uses four charge-storage capacitors to encode the signal phase, each for a quarter period of modulation frequency. (b) Maximum achievable SNR as a function of a pixel FWC. Left shaded area covers most common camera sensors. Right axis indicates the minimum ADC resolution needed to resolve pixel value without compromising SNR.

limit on the signal-to-noise ratio achievable for a given sensor design, independently of the optical imaging setup efficiency. Figure 2(b) illustrates the maximum achievable shot-noiselimited SNR for a given capacity of a sensor pixel. Typical camera sensors have a FWC in the range of $10^{4}-10^{5}$ corresponding to an SNR $\lesssim 300$. Sensors with the largest FWC are preferable for sensitive ODMR-based magnetometry, provided that the number of fluorescence photons impinging the camera during a single exposure time is sufficiently high.

\section{B. Camera frame rate}

For a given well capacity of a pixel, the number of photoelectrons collected per unit time can be maximized by running the camera at high frame rates (under sufficiently strong illumination). Given that the maximum number of frames that a camera is able to capture per second is FPS, the maximum number of photoelectrons recorded in $1 \mathrm{~s}$ is simply 
TABLE I. Camera sensor parameters used for determination of achievable SNR and magnetic field sensitivity.

\begin{tabular}{lccccccc}
\hline \hline Camera \# & Sensor type & $\begin{array}{c}\text { Pixel size } \\
(\mu \mathrm{m})\end{array}$ & $\begin{array}{c}\text { Array size } \\
\text { (pixels) }\end{array}$ & QE $(\%)$ & $\begin{array}{c}\text { FPS with } \\
\text { full resolution }\end{array}$ & $\begin{array}{c}\text { FWC } \\
\left(\mathrm{e}^{-}\right)\end{array}$ & $\begin{array}{c}\text { ADC } \\
(\text { bits })\end{array}$ \\
\hline 1 & CMOS & $5.3 \times 5.3$ & $1280 \times 1024$ & $\sim 60$ & 60 & $8 \times 10^{3}$ & 10 \\
2 & CMOS & $4.8 \times 4.8$ & $1280 \times 1024$ & $30-55$ & 224 & $1 \times 10^{4}$ & 10 \\
3 & EM-CCD & $16 \times 16$ & $512 \times 512$ & $>90$ & 56 & $1.8 \times 10^{5}$ & 16 \\
4 & CMOS & $128 \times 128$ & $128 \times 128$ & $\sim 50$ & 10000 & $1 \times 10^{8}$ & 14 \\
5 & Lock-in & $39.6 \times 39.6$ & $280 \times 292$ & $60-80$ & $3800^{\mathrm{a}}$ & $3.5 \times 10^{5}$ & $2 \times 10^{\mathrm{b}}$ \\
\hline \hline
\end{tabular}

Internally up to 1000000 FPS (modulation frequency $\leq 250 \mathrm{kHz}$ ).

${ }^{\mathrm{b}}$ Separate in-phase and quadrature output images.

given by $S_{p x}^{1 s}=$ FPS FWC. Although the camera has a certain dead (processing) time, it does not limit the sensitivity as long as there is enough light available for exposure. The bandwidth-normalized SNR is thus given by the following:

$$
\mathrm{SNR}_{p x, \max }^{1 s}=\sqrt{\mathrm{FPS} \cdot \mathrm{FWC}} .
$$

The above equation shows that the most suitable camera is the one with the largest product of the pixel capacity and the frame rate. For the deep-well CMOS sensor \#4 (see Table I), in order to achieve the maximum sensitivity, a pixel has to collect as many as $10^{12}$ photoelectrons per second, which amounts to $\sim 1 \mu \mathrm{W}$ of optical power delivered to a pixel and $\sim 10 \mathrm{~mW}$ in total for uniform sensor illumination. Although these are high fluorescence levels, even larger values from a quasi-2D NV layer have been reported in Ref. 25.

\section{Sensor resolution}

The most common laboratory cameras offer resolution in the megapixel range and use sensors of a $(1 / 3)^{\prime \prime}-1$ " size with a photosensitive area length of $\sim 5$ to $13 \mathrm{~mm}$. This implies a single pixel length in the order of $\sim 1$ to $10 \mu \mathrm{m}$. The larger the pixel size, the higher is its FWC and the more the number of photons that can be collected before saturation. As a consequence, sensors with the largest potential wells usually have a reduced spatial resolution, as summarized in Table I. However, high-resolution cameras offer the additional option to combine data from several small pixels to form a single "macro-pixel." This can be done through post-processing of image data, with an effective FWC of a macro-pixel being the sum of FWCs of constituting pixels, at the cost of having a larger amount of data required to be processed. Many sensors offer direct binned readout, e.g., \#1 and \#2. The on-sensor binning, however, does not improve the effective FWC.

Another important factor for accurate imaging is the digital resolution of the analog-to-digital converters (ADCs) in the camera. Entry-level cameras typically acquire images with a pixel resolution of 8-10 bit (for color cameras, separately for red, green, and blue components), while the scientificgrade cameras most often have a resolution of 12-16 bit per pixel. The ADC resolution is usually matching the ratio of the FWC to the electronic readout noise. This value defines the camera's dynamic range, i.e., ratio of the largest to the smallest detectable signal. However, in order to effectively use the large FWC for precision ODMR detection, the number of bits required to capture images with the maximum SNR is given by $\log _{2}\left(\mathrm{SNR}_{p x}^{\max }\right)=0.5 \log _{2}(\mathrm{FWC})$ and summarized in Fig. 2(b). All the cameras listed in Table I provide large enough $\mathrm{ADC}$ resolution for that purpose. Additional bits are useful for exploiting the full dynamic range of the sensor but come at the cost of a larger bandwidth requirement for continuous image data transfer or the necessity of an internal camera memory for burst-mode video capturing.

\section{Quantum efficiency and fill factor}

The number of photoelectrons collected by the camera forms a fraction of all the incoming photons due to the finite quantum (conversion) efficiency and a limited photo-active area within each pixel. Due to their design, CCD cameras offer almost unity FF [Fig. 2(a)] and highest QE, exceeding 90\% for back-illuminated sensors. On the other hand, most CMOS cameras have a limited FF due to the presence of several transistors in each pixel [Fig. 2(a)]. This is often mitigated by the inclusion of micro-lens arrays in CMOS cameras, which focus light onto the photo-active regions and lift the effective FF close to unity. For both the technologies, QE.FF exceeds $50 \%$ for the best cameras.

\section{E. In-pixel lock-in detection}

With the advent of modern CMOS fabrication processes, a new type of cameras became commercially available: the timeof-flight (ToF) cameras, having primary use in range-finding. In the simplest ToF sensor, the charge accumulated on a pixel photodiode is transferred by accurately timed gating electronics to one out of the two holding wells (capacitors). Arrival time of a light pulse may be then determined by comparing the number of photons (collected charges) that arrived before and after the trigger that switches the active capacitor. The concept of the ToF camera has been extended onto a phasesensitive demodulation. The charge from a pixel photodiode is transferred sequentially between four wells, as indicated in Fig. 2, with the cycle period matching the applied modulation frequency. ${ }^{26,27}$ In such a case, the voltage measured across the wells $I_{+}\left(Q_{+}\right)$and $I_{-}\left(Q_{-}\right)$represents an in-phase (quadrature) signal. Low-pass filtering is performed by accumulating the charges over many modulation periods before reading out the pixel value. This enables per-pixel lock-in demodulation of the optical signal, and it is the main operation principle behind camera \#5. This camera sensor can additionally, after each modulation cycle, perform the dc signal 
TABLE II. Maximum pixel SNR and the corresponding magnetic field sensitivity. Last column indicates the optical fluorescence power that is required to achieve $\delta B_{p x, \max }^{1 s}$ sensitivity over the full sensor area.

\begin{tabular}{lccccc}
\hline \hline Camera \# & $\mathrm{SNR}_{p x, \max }^{\exp }$ & $\delta B_{p x, \max }^{\exp }(\mathrm{nT})$ & $\delta B_{p x, \max }^{1 s}(\mathrm{nT})$ & $\delta B_{128 p x}^{1 s}(\mathrm{nT})$ & Optical power \\
\hline 1 & 89 & 7979 & 1030 & 129 & $0.3 \mu \mathrm{W}$ \\
2 & 100 & 7137 & 477 & 60 & $2.2 \mu \mathrm{W}$ \\
3 & 424 & 1682 & 225 & 56 & $0.9 \mu \mathrm{W}$ \\
4 & 10000 & 71 & 0.7 & 0.7 & $10 \mathrm{~mW}$ \\
5 & $66^{\mathrm{a}}$ & 539 & 8.8 & 4.0 & $1 \mathrm{~mW}$ \\
\hline \hline
\end{tabular}

${ }^{\mathrm{a}}$ Due to a different operation principle, a non-lock-in camera requires $\mathrm{SNR}_{p x, \max }^{\exp }=1323$ to achieve a similar field sensitivity.

(constant photocurrent) subtraction and, therefore, can more effectively use the FWC of a pixel. This will be further discussed below.

Assuming full amplitude modulation of the MW field, the ODMR signal consists of a steady fluorescence level $S$ and a modulated part of amplitude $C \cdot S$. The camera's FWC value shown in Table I refers to the maximum signal difference at the sense node ${ }^{28}$ (Fig. 2), while a single capacitor is about two times smaller. When the camera is operated in the dc-subtraction mode, the maximum charge that can be stored in a single capacitor is given by the relation: $C \cdot S=\mathrm{FWC} / 2$ or, equivalently, $S=\mathrm{FWC} / 2 C$. The latter form allows us to determine the optical shot-noise level to be $N=\sqrt{\mathrm{FWC} / 2 C}$, which includes the noise from photons corresponding to the subtracted dc part of the signal. The magnetic field sensitivity for such a camera is, therefore, given by the following:

$$
\delta B \simeq \frac{\hbar}{g \mu_{B}} \frac{\Gamma}{C \cdot S /(\sqrt{2} N)}=\frac{\hbar}{g \mu_{B}} \frac{\Gamma}{\sqrt{\mathrm{FWC} \cdot C / 4}},
$$

where the noise has been multiplied by factor of $\sqrt{2}$ that comes from the output signal being voltage-measured across 2 capacitors with similar noise. Equation (6) indicates that the lock-in camera offers a sensitivity improvement by a factor of $1 / \sqrt{4 C}$ over a non-lock-in sensor with an identical pixel FWC. This makes a marked difference for lowcontrast diamond samples. Additionally, the phase-sensitive detection reduces the technical noise, e.g., originating from laser light intensity fluctuations, by means of a common-mode rejection.

\section{F. Magnetic field sensitivity estimation}

Using the parameters of the cameras shown in Table I, Eq. (3), and the SNR formulas discussed above, we estimate the values of the smallest detectable magnetic field that can be resolved by each camera. We determine the sensitivity of a single pixel for a single frame and for $1 \mathrm{~s}$ of a combined exposure (using the maximum frame rate supported with full camera resolution). To account for the different resolution of the cameras, the latter sensitivity is also scaled to the effective resolution of $128 \times 128$ pixels, $\delta B_{128 p x}^{1 s}$. We assume here an ODMR contrast $C=5 \%$, a resonance width of $\Gamma / 2 \pi=1 \mathrm{MHz}$, and that the magnetic field is aligned with the NV axis. Results of the sensitivity estimation are shown in Table II together with the fluorescence light power required (assuming $\lambda=650 \mathrm{~nm}$ ) in order to sufficiently illuminate the full sensor.

\section{EXPERIMENTAL RESULTS}

Our diamond sensor is a $2 \times 2 \times 0.5 \mathrm{~mm}^{3}$ electronic-grade diamond substrate (Element 6 ), on top of which a ${ }^{12} \mathrm{C}$ isotopically purified and ${ }^{15} \mathrm{~N}$ nitrogen-rich layer $\left(\left[{ }^{12} \mathrm{C}\right]>99.99 \%\right.$, $\left[{ }^{15} \mathrm{~N}\right] \sim 10 \mathrm{ppm}, 1 \mu \mathrm{m}$ thickness) was grown using chemical vapor deposition (CVD). Vacancies were introduced by $1.8 \mathrm{MeV}$ helium ion implantation $\left(\sim 10^{15} \mathrm{~cm}^{-2}\right.$ dose $)$, followed by $2 \mathrm{~h}$ of annealing in a vacuum at $900{ }^{\circ} \mathrm{C}$. The resulting $\mathrm{NV}$ concentration is estimated to be in the order of 0.1-1 ppm.

Light is delivered to and collected from the diamond through the same microscope objective (Mitutoyo M Plan Apo NIR HR $100 \times$, NA $=0.7$ ) in an inverted microscope arrangement. The top diamond surface is coated with a 300-nm-thick aluminum layer to reflect the excitation and fluorescence light. The bottom side of the diamond is antireflection coated with silica, further aiding the light collection and reducing the interference within the diamond. Typically around $5 \mu \mathrm{W}$ of red fluorescence is detected when the $\mathrm{NV}$ centers are excited with $150 \mathrm{~mW}$ green excitation power (Verdi G8, $532 \mathrm{~nm}$ ). The illuminated area is imaged on a camera sensor with $45 \times$ magnification, leading to a pixel size of $\sim(0.9 \mu \mathrm{m})^{2}$ at the diamond plane. The actual resolution is lower (Airy disc radius of $\sim 7.5 \mu \mathrm{m}$ ) as the objective does not compensate for spherical aberrations introduced by imaging through the 500- $\mu \mathrm{m}$-thick diamond. MWs were generated with a signal generator (Stanford Research Systems SG394) and on-off keyed with a switch (Mini-Circuits ZASWA-250DR+). The lock-in camera (Heliotis heliCam C3) provided a square-wave modulation signal with a frequency set to $3.7 \mathrm{kHz}$. After passing the switch, MWs are amplified by a high-power amplifier (Mini-Circuits, ZHL-16W-43+) and delivered to the printed circuit-board antenna ${ }^{29}$ placed under the 3D-printed diamond holder with a distance of $1 \mathrm{~mm}$ between the copper trace and the NV sensing layer. A pair of permanent magnets created a uniform magnetic field of around $2 \mathrm{mT}$ parallel to the diamond surface and aligned with the [110] crystal direction.

Figure 3 shows the results of fluorescence and ODMR measurements using camera \#5. All the images were captured in full resolution; however, only the $100 \times 100$ pixels region with the illuminated spot is shown. The fluorescence profile recorded with the camera operating in the intensity mode, i.e., when no phase-sensitive detection is performed, is shown in Fig. 3(a). The deviation from Gaussian-shape results from the imperfection of the excitation beam-profile as well as 

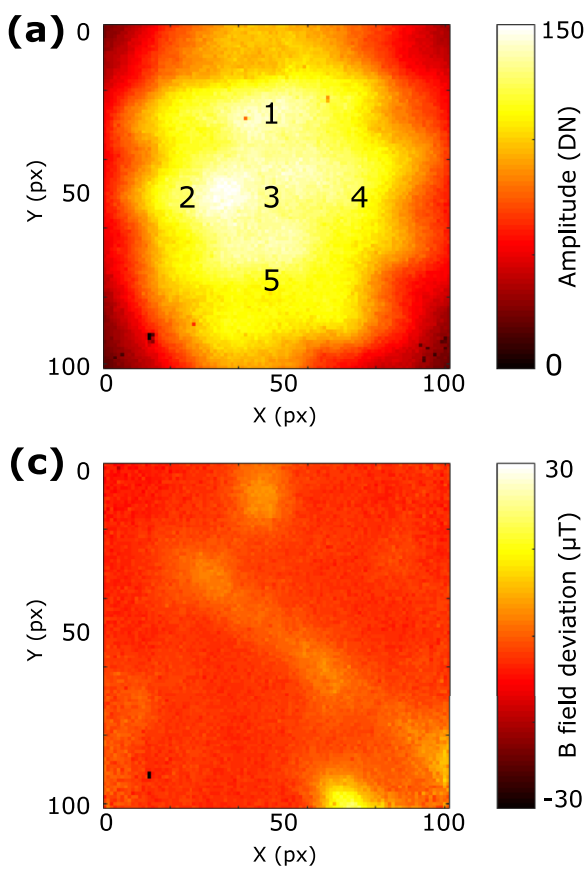
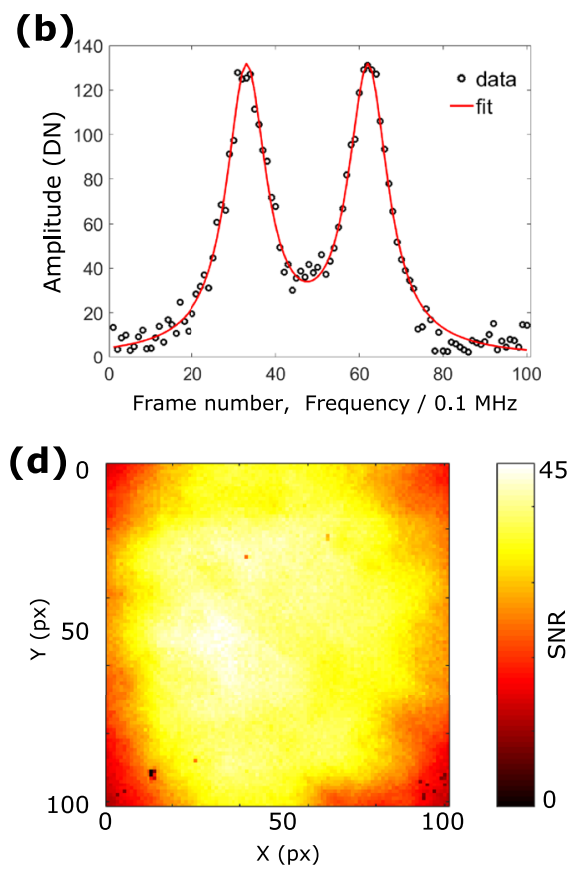

FIG. 3. Magnetic field imaging using the lock-in camera. (a) $100 \times 100$ pixel fluorescence intensity profile. $\mathrm{DN}$ refers to data number (ADC units). (b) Central-pixel ODMR spectrum taken from 100 frames captured during 10-MHz-wide MW sweep in a uniform magnetic field. (c) Relative position of the resonance frequency determined from the fits and plotted in magnetic field units. (d) SNR profile calculated from the fit parameters and noise amplitude measured without MWs. non-uniform nitrogen incorporation during the CVD growth of the sensing layer and residual interference within the diamond. Five points in a cross-like arrangement around the center were selected for the sensitivity analysis as indicated in Fig. 3(a).

For the phase-sensitive demodulation, the camera was set to accumulate the signal over 62 modulation periods with background subtraction enabled, resulting in a frame rate of FPS $=$ 59.7. ODMR spectra were recorded by sweeping the MW frequency over a $10 \mathrm{MHz}$ range synchronously with the acquisition of $2 \times 100$ frames, where the factor of 2 comes from simultaneous acquisition of in-phase (I) and quadrature (Q) images. The two quadratures were subsequently combined for each (x,y) pixel via $S(x, y)=\sqrt{I(x, y)^{2}+Q(x, y)^{2}}$. Figure 3(b) shows the ODMR resonance data recorded at the central point, $\mathrm{S}(50,50)$. Two hyperfine resonances separated by $3 \mathrm{MHz}$ are visible, corresponding to the left pair of resonances shown in Fig. 3(b). The phase-sensitive demodulation of the signal results in zero background. In our case, however, the signal is calculated from both the quadratures and the presence of noise translates into a small background shift. For each pixel, a double-Lorentzian curve was fitted, assuming the same amplitude and width for both peaks. The result of a fit for the central pixel is also shown in Fig. 3(b). The resonance amplitudes at points 1-5, extracted from the fit parameters, were equal to $142.4,126.4,133.0,122.8$, and 116.1 , respectively. Spectral position of the lower frequency peak, determined from the fit, was used to calculate the magnetic field variation across the full image. The resulting field map is shown in Fig. 3(c) and it is in agreement with a diamond being placed in a uniform magnetic field. Only small $(<5 \mu \mathrm{T})$ variations are visible in the image, except for a larger deviation $(\sim 15 \mu \mathrm{T})$ spot at the bottom, where, however, the fluorescence intensity is low.

To quantify the SNR in the recorded data, an additional set of images were taken in the absence of MWs. These were similarly converted to amplitude images, and the standard deviation across 200 frames was calculated independently for each pixel. Fitted ODMR peak amplitudes and noise data were then used to create the SNR map shown in Fig. 3(d). In the central region, the SNR exceeds 40 , which translates into $1 \mu \mathrm{T}$ magnetic sensitivity in a single frame and $142 \mathrm{nT}$ when all the frames acquired within $1 \mathrm{~s}$ are averaged (combined exposure). The latter sensitivity value is limited by the frame rate at which we operate the camera and, in principle, could be improved by up to 8 times if more fluorescence light was available.

When the spatial resolution of a camera can be sacrificed, additional gain in the sensitivity can be achieved by means of a binning of the pixel data. The amplitudes extracted from the fits described above were summed over a $k \times k$ pixel region around the points labeled in Fig. 3, and the corresponding noise level was calculated from the data in the absence of MWs. The resulting SNR is shown in Fig. 4(a). As can be expected for white uncorrelated noise, the SNR scales linearly with the macro-pixel length (square root of the area). For the bin size of 49 pixels, a sensitivity of $\sim 20 \mathrm{nT}$ is achieved, which corresponds to $\sim 2.6 \mathrm{nT}$ for $1 \mathrm{~s}$ of a combined exposure. In order to compare this sensitivity value with other $\mathrm{NV}$ experiments, we assume the Nyquist bandwidth of FPS/2, which yields the bandwidth-normalized sensitivity of $\sim 3.7 \mathrm{nT} \mathrm{Hz}^{-1 / 2}$. An independent measurement using a photodiode and a standard lock-in amplifier yields a similar sensitivity of $\sim 1.5 \mathrm{nT} \mathrm{Hz}^{-1 / 2}$ of our setup, albeit all the available light was collected by the diode. The SNR normalized to the bin size is shown in Fig. 4(b). The initial values (for low $k$ ) depend heavily on the pixel choice as the recorded intensity profile is not uniform. In one region, an SNR of $>40$ is maintained over a $20 \times 20$ pixel area. As the binning window size is increased, all the values converge around 35 . For $k>25$, the binning areas partially overlap, resulting in a further loss of spatial resolution. 
(a)

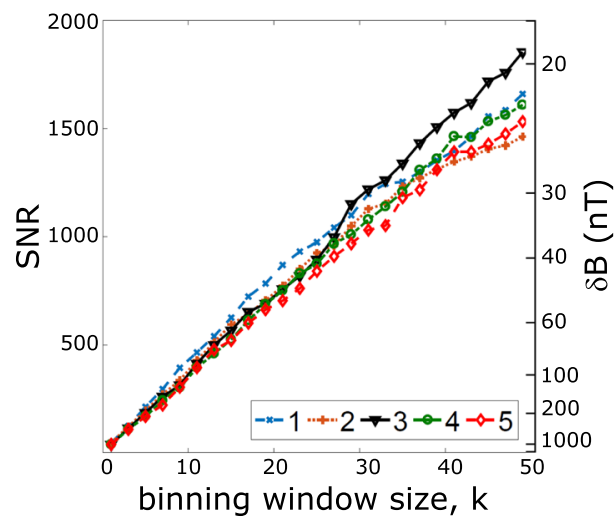

(b)

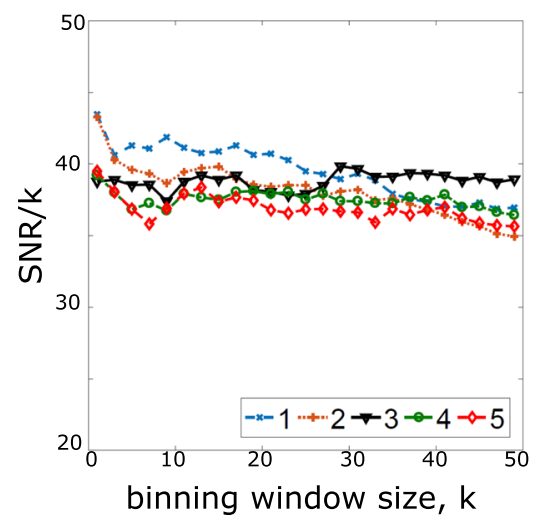

FIG. 4. (a) SNR calculated for macropixels consisting of $\mathrm{k} \times \mathrm{k}$ pixels around the positions indicated by labels $1-5$ in Fig. 3. (b) SNR data normalized to the window size. For small bins, the SNR is determined by the intensity profile.

\section{SUMMARY AND CONCLUSIONS}

Several conclusions can be drawn from our analysis which is summarized in Table II. First, for standard CCD/CMOS sensors $\left(\mathrm{FWC} \sim 10^{4}-10^{5}\right.$ ) and a sample contrast of $\mathrm{C} \sim 1 \%$, the signal-to-noise ratio multiplied by the ODMR contrast is on the order of unity. This limits the single-exposure sensitivity $\delta B_{p x, \max }^{\exp }$ to be a large fraction of the resonance linewidth ( $\sim 40 \mathrm{~T}$ ). Higher sensitivity may only be achieved through the temporal averaging, especially with fast frame rate cameras, and/or through binning the captured images which reduces the spatial resolution. On the other hand, deep-well sensors achieve much higher sensitivity by allowing measurements of small relative fluorescence changes $\Delta F / F \sim 10^{-4}$ when sufficient amount of light is available. The required fluorescence levels were recently reported in Ref. 25 for a $200 \times 2000$ $\times 13 \mu \mathrm{m}^{3}$ size NV layer with a similar density and resonance linewidth as in our sample.

The lock-in sensors are an interesting choice for ODMR imaging for multiple reasons: the dc subtraction mechanism allows for efficient usage of the pixel well capacity; the pixel value after background subtraction may be directly proportional to the field change; and the technical noise due to the laser instability is easily suppressed. The latter becomes increasingly important when smaller fluorescence changes are to be measured at higher frame rates. Additionally, cameras using phase-sensitive detection may find their application in alkali-vapor magnetometry through the detection of the Larmor precession frequency ${ }^{30}$ and for a high resolution and bandwidth motion detection of objects trapped, for instance, in optical tweezers.

We have experimentally demonstrated the single-pixel and single-frame sensitivity of around $1 \mu \mathrm{T}$ while maintaining acquisition speed of 60 frames/s. There are several ways in which the sensitivity of our setup can be further improved. First, by adjusting the demodulation phase such that only in-phase or quadrature image would be needed. This can be accomplished by using an external demodulation reference and would result in a $\sqrt{2}$ sensitivity improvement. Second, the camera frame rate can be increased up to 64 times. This requires an improved fluorescence collection and would result in a further 8-fold improvement of the bandwidth-normalized sensitivity. Finally, multiple successive frames or nearby pixel values can be averaged to further increase the sensitivity, at a cost of reduced spatial or temporal resolution.

In conclusion, we have discussed the main cameraimposed limitations for precision, wide-field NV ODMR detection, which enables sensitive imaging of the magnetic field, temperature, electric field, or pressure. The presented analysis applies also to high-pressure, high-temperature diamonds and other systems exhibiting low fluorescence contrast like, for example, silicon vacancy centers in silicon carbide. ${ }^{31}$ With NV centers in diamond, we have demonstrated that nanotesla-sensitivity magnetometry at video frame rates is possible. Such imaging systems could be useful for the sensitive monitoring of small field changes in real-time and, with high spatial resolution, in opening new possibilities for studying dynamical systems, e.g., for motion tracking of magnetically tagged particles.

\section{ACKNOWLEDGMENTS}

We thank Kristian Hagsted Rasmussen for coating the diamond. We acknowledge stimulating discussions with Haitham El-Ella, Sepehr Ahmadi, and Axel Thielscher. This work was supported by the Innovation Fund Denmark (EXMAD and QUBIZ projects), Novo Nordisk Foundation, IQst, DFG, VW Stiftung, ERC, BMBF, and EU DIADEMS.

${ }^{1}$ F. Jelezko, I. Popa, A. Gruber, C. Tietz, J. Wrachtrup, A. Nizovtsev, and S. Kilin, Appl. Phys. Lett. 81, 2160 (2002).

${ }^{2}$ M. W. Doherty, N. B. Manson, P. Delaney, F. Jelezko, J. Wrachtrup, and L. C. L. Hollenberg, Phys. Rep. 528, 1 (2013); e-print arXiv:1302. 3288.

${ }^{3}$ G. Kucsko, P. C. Maurer, N. Y. Yao, M. Kubo, H. J. Noh, P. K. Lo, H. Park, and M. D. Lukin, Nature 500, 54 (2013); e-print arXiv:1304.1068.

${ }^{4}$ F. Dolde, H. Fedder, M. W. Doherty, T. Noebauer, F. Rempp, G. Balasubramanian, T. Wolf, F. Reinhard, L. C. L. Hollenberg, F. Jelezko, J. Wrachtrup, and T. Nöbauer, Nat. Phys. 7, 459 (2011); e-print arXiv:1103.3432.

${ }^{5}$ L. Rondin, J.-P. Tetienne, T. Hingant, J.-F. Roch, P. Maletinsky, and V. Jacques, Rep. Prog. Phys. 77, 056503 (2014); e-print arXiv:1311. 5214.

${ }^{6}$ J. R. Maze, P. L. Stanwix, J. S. Hodges, S. Hong, J. M. Taylor, P. Cappellaro, L. Jiang, M. V. G. Dutt, E. Togan, A. S. Zibrov, A. Yacoby, R. L. Walsworth, and M. D. Lukin, Nature 455, 644 (2008).

${ }^{7}$ J. R. Rabeau, P. Reichart, G. Tamanyan, D. N. Jamieson, S. Prawer, F. Jelezko, T. Gaebel, I. Popa, M. Domhan, and J. Wrachtrup, Appl. Phys. Lett. 88, 023113 (2006); e-print arXiv:0511722 [cond-mat]. 
${ }^{8}$ S. Pezzagna, B. Naydenov, F. Jelezko, J. Wrachtrup, and J. Meijer, New J. Phys. 12, 065017 (2010).

${ }^{9}$ L. M. Pham, D. Le Sage, P. L. Stanwix, T. K. Yeung, D. Glenn, A. Trifonov, P. Cappellaro, P. R. Hemmer, M. D. Lukin, H. Park, A. Yacoby, and R. L. Walsworth, New J. Phys. 13, 045021 (2011); e-print arXiv: 1207.3339.

${ }^{10} \mathrm{CCD}$-charged coupled device, CMOS-complementary metal-oxide.

${ }^{11}$ M. Chipaux, A. Tallaire, J. Achard, S. Pezzagna, J. Meijer, V. Jacques, J.-F. Roch, and T. Debuisschert, Eur. Phys. J. D 69, 166 (2015); e-print arXiv: 1410.0178 .

${ }^{12}$ D. Le Sage, K. Arai, D. R. Glenn, S. J. DeVience, L. M. Pham, L. Rahn-Lee, M. D. Lukin, A. Yacoby, A. Komeili, and R. L. Walsworth, Nature 496, 486 (2013).

${ }^{13}$ D. A. Simpson, J.-P. Tetienne, J. M. McCoey, K. Ganesan, L. T. Hall, S. Petrou, R. E. Scholten, and L. C. L. Hollenberg, Sci. Rep. 6, 22797 (2016); e-print arXiv:1508.02135.

${ }^{14}$ J. Smits, A. Berzins, F. H. Gahbauer, R. Ferber, K. Erglis, A. Cebers, and J. Prikulis, Eur. Phys. J. Appl. Phys. 73, 20701 (2016).

${ }^{15}$ A. Nowodzinski, M. Chipaux, L. Toraille, V. Jacques, J.-F. Roch, and T. Debuisschert, Microelectron. Reliab. 55, 1549 (2015); e-print arXiv:1512.01102.

${ }^{16}$ J.-P. Tetienne, N. Dontschuk, D. A. Broadway, A. Stacey, D. A. Simpson, and L. C. L. Hollenberg, Sci. Adv. 3, e1602429 (2017); e-print arXiv:1609.09208.

${ }^{17}$ D. R. Glenn, R. R. Fu, P. Kehayias, D. Le Sage, E. A. Lima, B. P. Weiss, and R. L. Walsworth, Geochem., Geophys., Geosyst. 18, 3254, https://doi.org/ 10.1002/2017gc006946 (2017); e-print arXiv:1707.06714.

${ }^{18}$ R. Homma, B. J. Baker, L. Jin, O. Garaschuk, A. Konnerth, L. B. Cohen, and D. Zecevic, Philos. Trans. R. Soc., B 364, 2453 (2010).

${ }^{19}$ J. H. Park, J. Platisa, J. V. Verhagen, S. H. Gautam, A. Osman, D. Kim, V. A. Pieribone, and E. Culurciello, J. Neurosci. Methods 201, 290 (2011).
${ }^{20}$ S. Beer, P. Zeller, N. Blanc, F. Lustenberger, and P. Seitz, "Smart pixels for real-time optical coherence tomography," Proc. SPIE 5302, 21-32 (2004).

${ }^{21}$ A. M. Edmonds, U. F. S. D'Haenens-Johansson, R. J. Cruddace, M. E. Newton, K. M. C. Fu, C. Santori, R. G. Beausoleil, D. J. Twitchen, and M. L. Markham, Phys. Rev. B 86, 035201 (2012); e-print arXiv:1112. 5757.

${ }^{22}$ M. Lesik, J. P. Tetienne, A. Tallaire, J. Achard, V. Mille, A. Gicquel, J. F. Roch, and V. Jacques, Appl. Phys. Lett. 104, 113107 (2014); e-print arXiv: 1401.2795

${ }^{23}$ V. M. Acosta, E. Bauch, M. P. Ledbetter, C. Santori, K.-M. M. C. Fu, P. E. Barclay, R. G. Beausoleil, H. Linget, J. F. Roch, F. Treussart, S. Chemerisov, W. Gawlik, and D. Budker, Phys. Rev. B 80, 115202 (2009); e-print arXiv:0903.3277.

${ }^{24}$ P. C. Maurer, G. Kucsko, C. Latta, L. Jiang, N. Y. Yao, S. D. Bennett, F. Pastawski, D. Hunger, N. Chisholm, M. Markham, D. J. Twitchen, J. I. Cirac, and M. D. Lukin, Science 336, 1283 (2012); e-print arXiv: $1202.4379 \mathrm{v} 1$.

${ }^{25}$ J. F. Barry, M. J. Turner, J. M. Schloss, D. R. Glenn, Y. Song, M. D. Lukin, H. Park, and R. L. Walsworth, Proc. Natl. Acad. Sci. U. S. A. 113, 14133 (2016); e-print arXiv: 1602.01056

${ }^{26}$ T. Spirig and P. Seitz, IEEE Trans. Electron Devices 44, 1643 (1997).

${ }^{27}$ T. Oggier, M. Lehmann, R. Kaufmann, M. Schweizer, M. Richter, and P. Metzler, Proc. SPIE 5249, 534 (2004).

${ }^{28}$ A. G. Heliotis, Switzerland, private communication (2016).

${ }^{29}$ S. Ahmadi, H. A. R. El-Ella, J. O. B. Hansen, A. Huck, and U. L. Andersen, Phys. Rev. Appl. 8, 034001 (2017); e-print arXiv:1707.00502.

${ }^{30}$ S. Pustelny, A. Wojciechowski, M. Gring, M. Kotyrba, J. Zachorowski, and W. Gawlik, J. Appl. Phys. 103, 063108 (2008); e-print arXiv:0708. 3488.

${ }^{31}$ D. Simin, F. Fuchs, H. Kraus, A. Sperlich, P. G. Baranov, G. V. Astakhov, and V. Dyakonov, Phys. Rev. Appl. 4, 014009 (2015); e-print arXiv:1505. 00176 